\title{
Autoimmune Cholangiopathy followed with the Diagnosis of Cholangiocarcinoma
}

\author{
Ozlem Ozer Cakir, Huseyin Ataseven, Murat Biyik, Gokhan Gungor, Ali Demir
}

\begin{abstract}
Autoimmune cholangiopathy (IgG4-associated sclerosing cholangitis) can mimic symptoms and signs of many other diseases and thus it remains often undiagnosed or misdiagnosed. The definitive diagnosis is based on pathological findings. Thus, only very few patients have been reported in the literature. We report a case of a 64-year-old female with autoimmune cholangiopathy (IgG4-associated sclerosing cholangitis) that had presented mass-like lesions at liver parenchyma and occlusion of the common bile duct and performed a metal stent inserted in the common bile duct for diagnosis of cholangiocarcinoma in another medical center 5 years ago. High levels of the IgG4 of the patient and pathologic findings of mass-like lesion specimen disclosed severe infiltrations of lymphocytes and plasma cells and suggested an autoimmune cholangiopathy (IgG4-associated cholangiopathy). The patient was treated with prednisolone. The narrowing of the common bile duct, mass-like lesions at liver parenchyma and complaints of the patient improved significantly 1 month after corticosteroid treatment.
\end{abstract}

Abbreviations: MRCP: Magnetic resonance cholangiopancreatography; CT: Computed tomography; IPT: Inflammatory pseudotumor.

Keywords: Autoimmune cholangiopathy, IgG4-associated sclerosing cholangitis, Cholangiocarcinoma.

How to cite this article: Cakir OO, Ataseven H, Biyik M, Gungor G, Demir A. Autoimmune Cholangiopathy followed with the Diagnosis of Cholangiocarcinoma. Euroasian J HepatoGastroenterol 2012;2(2):119-121.

Source of support: Nil

Conflict of interest: None

\section{INTRODUCTION}

Cholangiocarcinoma is the second most common primary malignant hepatobiliary neoplasm, accounting for approximately $15 \%$ of liver cancers. Diagnosis of cholangiocarcinoma is challenging and the prognosis is poor, with recurrence rates of 60 to $90 \%$ after surgical resection. A wide spectrum of neoplastic and nonneoplastic conditions of the biliary tract may masquerade as cholangiocarcinoma, adding to the complexity of management in patients suspected to have cholangiocarcinoma. Mimics of cholangiocarcinoma constitute a heterogeneous group of entities that includes primary sclerosing cholangitis, recurrent pyogenic cholangitis, acquired immunodeficiency syndrome cholangiopathy, autoimmune pancreatitis, inflammatory pseudotumor, Mirizzi syndrome, xanthogranulomatous cholangitis, sarcoidosis, chemotherapy-induced sclerosis, hepatocellular carcinoma, metastases, melanoma, lymphoma, leukemia and carcinoid tumors. ${ }^{1}$

We report a case of autoimmune cholangiopathy (IgG4 associated sclerosing cholangitis) that had presented with mass-like lesions at liver parenchyma and occlusion of the common bile duct and performed a metal stent inserted in the common bile duct for diagnosis of cholangiocarcinoma in another medical center 5 years ago.

\section{CASE REPORT}

A 64-year-old woman was admitted to our hospital with a complaint of right upper abdominal pain and flatulence. The metal stent was inserted in the common bile duct for cholangiocarcinoma diagnosis at outside the center 5 years ago. The medical history was significant for a cholecystectomy and a splenectomy operation performed 10 and 26 years prior to presentation respectively. She was using insulin for type 2 diabetes mellitus for 6 years. In physical examination, conjunctivas were subicteric, a paramedian incisional scar was seen and sensitivity was present on palpation. The liver was palpable $2 \mathrm{~cm}$ below costal margine.

Her laboratory tests showed the following: Hemoglobulin: $10.7 \mathrm{gm} / \mathrm{dl}$; total bilirubin: $1.7 \mathrm{mg} / \mathrm{dl}$ (normal: 0.4-1.4); direct bilirubin: $0.5 \mathrm{mg} / \mathrm{dl}$ (normal: 0.1-0.5); total protein: 8.26 gm/dl (normal: 6.4-8.3); albumin: 3.5 gm/dl (normal: 3.5-5.0); alkaline phosphatase: $476 \mathrm{U} / \mathrm{l}$ (normal: 40-150); serum aspartate aminotransferase: $31 \mathrm{U} / \mathrm{l}$ (normal: 15-41); serum alanine aminotransferase, $27 \mathrm{U} / \mathrm{l}$ (normal: 14-54); and serum $\gamma$-glutamyltransferase: $227 \mathrm{U} / 1$ [normal: 7-50 (for women)]; erythrocyte sedimentation rate: $120 \mathrm{~mm} / \mathrm{h}$ (normal: 0-20). Basophilia was seen on peripheral blood smear.

Virologic markers, brucella and salmonella was negative. Vitamin $B_{12}$, ferritin, folic acid, thyroid stimulating hormone was normal. Ultrasonography of upper abdomen showed the liver larger than normal size $(17 \mathrm{~cm})$, the borders of heterogeneous mass lesion size was observed undistinguishable at the right liver lobe. Intrahepatic bile ducts were dilated.

Magnetic resonance cholangiopancreatography (MRCP) showed multifocal strictures, segmental ectasias, ductal wall thickening and irregular beading of intrahepatic and extrahepatic bile ducts (Fig. 1). Computerized tomography 


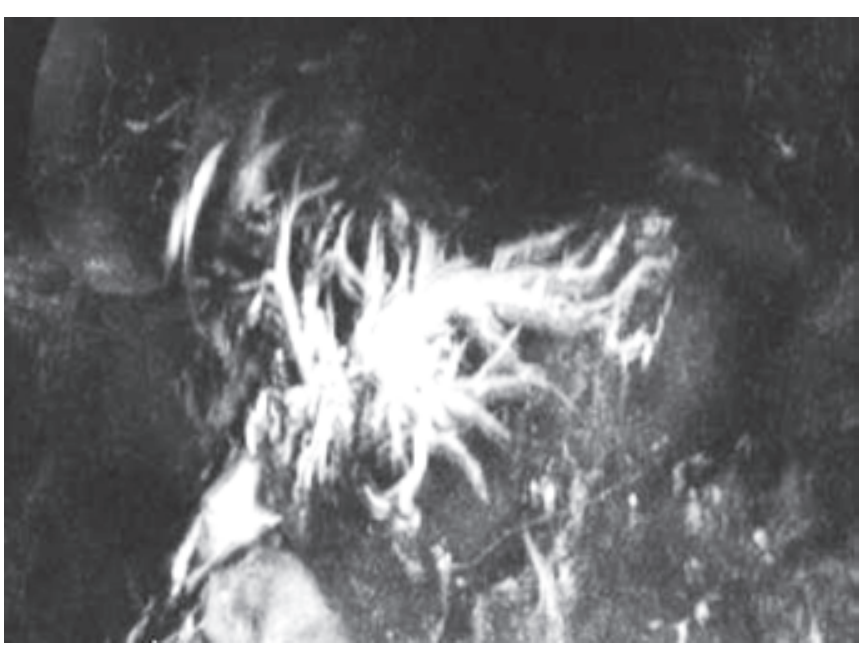

Fig. 1: MRCP showing multifocal strictures, segmental ectasias, ductal wall thickening and irregular beading of the intrahepatic and extrahepatic bile ducts

(CT) of the abdomen showed two hypodense mass-like lesion at liver parenchyma, dilatation of intrahepatic bile ducts (Fig. 2).

We performed the biopsy from mass-like lesion at liver parenchyma. The histopathologic examination of the lesion biopsy specimen showed fibrotic changes and inflammatory infiltration, rich in polymorphonuclear leukocyte, eosinophils, plasmocyte and histiocytes (Fig. 3).

Tumor markers were normal. Antismooth muscle antibody, antimicrosomal antibody, anti liver kidney muscle antibody was negative. Antinuclear antibody was positive.

The serologic tests showed the following: IgG: $25.2 \mathrm{gm} / \mathrm{l}$ (7.51-15.6 gm/l); IgG4: 1,320 mg/l (39.2-864 mg/l); IgE: 5 $\mathrm{IU} / \mathrm{ml}$ (0-165 IU/ml); and IgA: $5.02 \mathrm{gm} / \mathrm{l}(0.82-4.53 \mathrm{gm} / \mathrm{l})$.

Endoscopy of upper gastrointestinal system showed eosophageal varices grade 2, bile reflux gastropathy. Endoscopic retrograde cholangiopancreatography (ERCP)

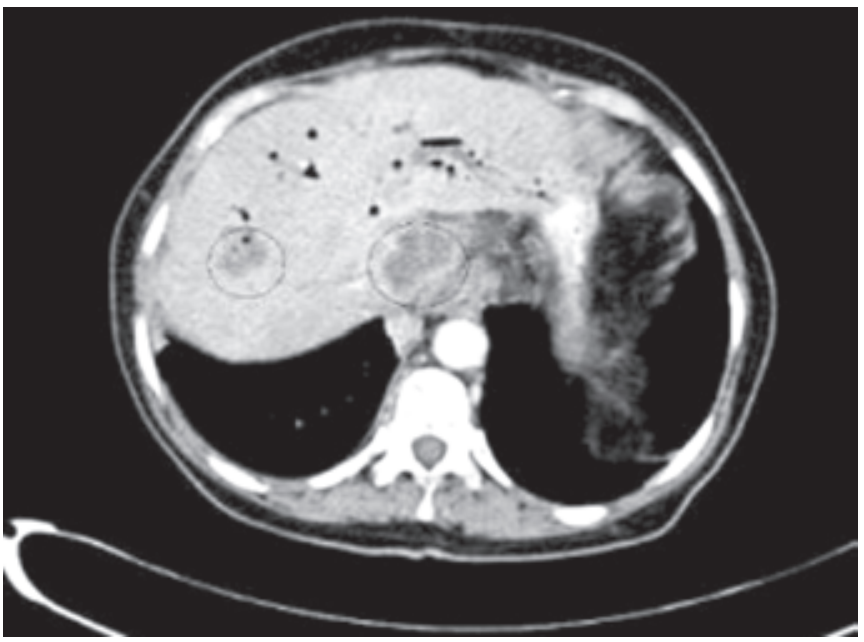

Fig. 2: CT showing two hypodense mass-like lesions at liver parenchyma

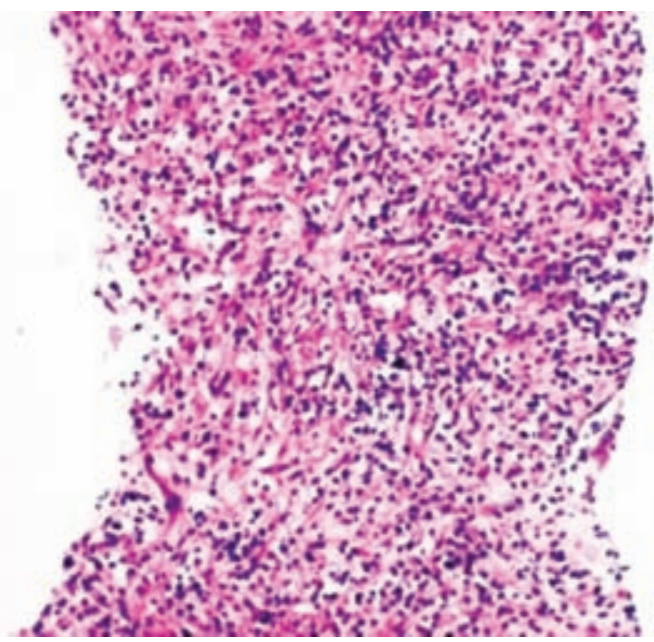

Fig. 3: Microscopic examination of liver lesion biopsy showing inflammatory infiltration rich in polymorphonuclear leukocyte, eosinophils, plasmocyte and histiocytes (hematoxylin eosin, $\times 40$ )

showed there was a metalic stent at the common bile duct and occlusion of common bile duct due to tissue infiltration into the metalic stent and balloon dilatation of common bile duct was performed. Then, we performed brush cytology from the common bile duct (Fig. 4). The cytologic examination of the common bile duct showed suspicious cells.

High levels of the cholestatic enzymes and IgG4 of the patient, a positive ANA test result, pathologic findings of mass-like lesion with severe infiltration of lymphocytes and plasma cells suggest an autoimmune cholangiopathy (IgG4 associated cholangiopathy).

After providing written informed consent, the patient was treated with $60 \mathrm{mg}$ of prednisolone daily and $15 \mathrm{mg}$ per $\mathrm{kg}$ of ursodeoxycholic acid daily for 4 weeks, prednisolone was reduced by $5 \mathrm{mg}$ biweekly until reaching $10 \mathrm{mg} /$ day. The narrowing of the common bile duct and

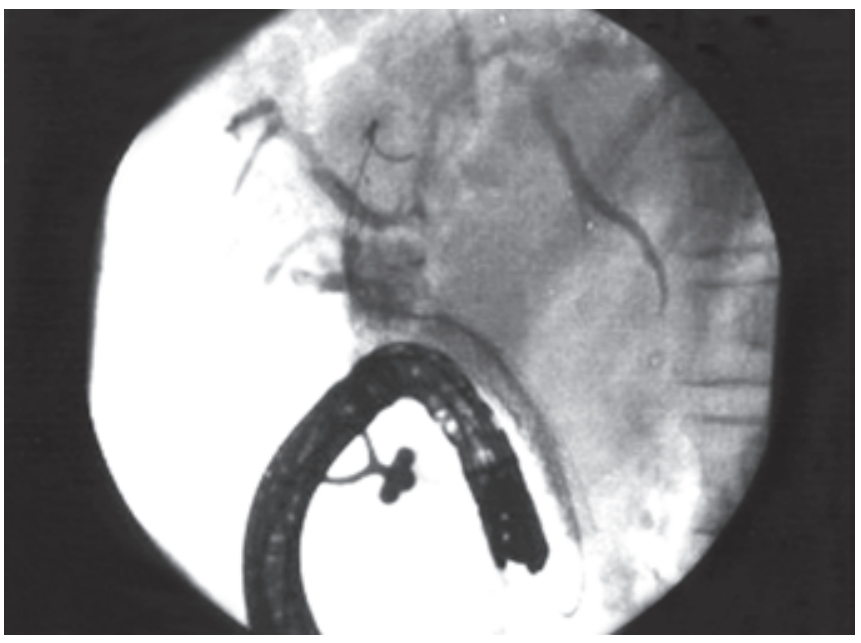

Fig. 4: ERCP showing a metalic stent at the common bile duct and occlusion of common bile duct due to tissue infiltration into the metalic stent 
complaints of the patient improved significantly after corticosteroid treatment.

\section{DISCUSSION}

Mimics of cholangiocarcinoma constitute a heterogeneous group of entities that includes primary sclerosing cholangitis, recurrent pyogenic cholangitis, acquired immunodeficiency syndrome cholangiopathy, autoimmune pancreatitis, inflammatory pseudotumor (IPT), Mirizzi syndrome, xanthogranulomatous cholangitis, sarcoidosis, chemotherapyinduced sclerosis, hepatocellular carcinoma, metastases, melanoma, lymphoma, leukemia and carcinoid tumors.

These entities demonstrate characteristic histomorphology and variable clinicobiologic behaviors. The imaging findings of these disparate entities may be indistinguishable from those of cholangiocarcinoma. In most cases, a definitive diagnosis can be established only with histopathologic examination of a biopsy specimen ${ }^{1}$ Autoimmune cholangiopathy is a recently proposed entity that describes a specific group of patients presenting overlapping features of primary biliary cirrhosis and autoimmune hepatitis, i.e. clinical and/or biochemical cholestasis, high titer antinuclear antibody, negative antimitochondrial antibody and elevated immunoglobulin G. Liver histology shows primary biliary cirrhosis coexisting with varying degrees of parenchymal inflammation. In addition, these patients achieve remission on corticosteroid therapy. The patient in this report fulfilled the above criteria. ${ }^{2-6}$

Since malignant tumors are frequently suspected on initial presentation, IgG4-related sclerosing disease should be considered in the differential diagnosis to avoid unnecessary surgery. ${ }^{2}$ IPT of the liver is a benign condition with a good prognosis. However, it is difficult to distinguish clinical and radiological findings of IPT from those of malignancies. Although most IPTs can be resolved with conservative therapy, surgical resection should be considered in cases of uncertain biopsy result, presumed malignant lesion, combination with other pathology or lack of response to conservative management. ${ }^{7}$

After providing written informed consent, the patient was treated with prednisolone and ursodeoxycholic acid and then the narrowing of the common bile duct and complaints of the patient improved significantly after corticosteroid treatment.

\section{REFERENCES}

1. Menias CO, Surabhi VR, Prasad SR, Wang HL, Nara VR, Chintapali KN. Mimics of cholangiocarcinoma: Spectrum of disease. Radiographics 2008;28:1115-29.

2. Kamisawa T, Okamoto A. IgG4-related sclerosing disease. World J Gastroenterol 2008;14:3948-55.

3. Brunner G, Klinge O. Ein der chronisch-destruierenden nichteitrigen Cholangitis ahnliches Krankheitsbild mit Antikörpern (Immuncholangitis). Dtsch Med Wochenschr 1987; 112:1454-55.

4. Ben-Ari Z, Dhillon AP, Sherlock S. Autoimmune cholangiopathy: Part of the spectrum of autoimmune chronic active hepatitis. Hepatology 1993;18:10-15.

5. Colombato LA, Alvarez F, Cote J, Huet PM. Autoimmune cholangiopathy: The result of consecutive primary biliary cirrhosis and autoimmune hepatitis? Gastroenterology 1994;107:1839-43.

6. Michieletti P, Wanless IR, Katz A, et al. Antimitochondrial antibody negative primary biliary cirrhosis — a distinct syndrome of autoimmune cholangitis. Gut 1994;35:260-65.

7. Ahn KS, Kang KJ, Kim YH, et al. Inflammatory pseudotumors mimicking intrahepatic cholangiocarcinoma of the liver; IgG4positivity and its clinical significance. J Hepatobiliary Pancreat Sci 2012;19:405-12.

\section{ABOUT THE AUTHORS}

\section{Ozlem Ozer Cakir (Corresponding Author)}

Department of Gastroenterology and Hepatology, Meram School of Medicine, Konya University, Konya, Turkey, e-mail: tansozlem@yahoo.com

\section{Huseyin Ataseven}

Department of Gastroenterology and Hepatology, Meram School of Medicine, Konya University, Konya, Turkey

\section{Murat Biyik}

Department of Gastroenterology and Hepatology, Meram School of Medicine, Konya University, Konya, Turkey

\section{Gokhan Gungor}

Department of Gastroenterology and Hepatology, Meram School of Medicine, Konya University, Konya, Turkey

\section{Ali Demir}

Department of Gastroenterology and Hepatology, Meram School of Medicine, Konya University, Konya, Turkey 